\title{
A Particle Swarm Optimization Algorithm for Finding DNA Sequence Motifs
}

\author{
Chengwei Lei and Jianhua Ruan \\ Department of Computer Science \\ The University of Texas at San Antonio \\ One UTSA Circle, San Antonio, TX 78249, USA \\ \{clei,jruan\}@cs.utsa.edu
}

\begin{abstract}
Discovering short DNA motifs from a set of co-regulated genes is an important step towards deciphering the complex gene regulatory networks and understanding gene functions. Despite significant improvement in the last decade, it still remains one of the most challenging problems in both computer science and molecular biology. In this work, we propose a novel motif finding algorithm based on a population-based stochastic optimization technique called Particle Swarm Optimization (PSO), which has been shown to be effective in optimizing difficult multidimensional problems in many fields. However, PSO has mainly been applied to problems in continuous domains. The motif finding problem, which is essentially a multiple local alignment problem, is discrete, as a slight shift in one sequence completely changes the alignment. Therefore, we propose to use a word dissimilarity graph to remap the neighborhood structure of the solution space, which transforms the motif finding problem into a contiguous integer domain, and propose a modification of the naive PSO algorithm to accommodate integer variables. In order to improve efficiency, we also propose several strategies for escaping from local optima, and determining the termination criteria automatically. Experimental results on simulated challenge problems show that our method is both more efficient and more accurate than several existing algorithms. Applications to several sets of real promoter sequences also show that our approach is able to detect known transcription factor binding sites, and outperforms two of the most successful existing algorithms.
\end{abstract}

\section{Introduction}

One of the most important mechanisms to regulate gene functions is on the transcription level, where the expression of genes is mediated by the binding of transcription factors (TF) to the promoter sequences of genes. Identifying common transcription factor binding sites (TFBS) from a set of putatively co-regulated genes is an important step towards deciphering the complex gene regulatory networks and understanding the tissue/condition-specific functions of genes.

The existing motif finding algorithms often differ from one another in their ways of defining motifs, the objective functions for calculating motif significance, and the search techniques used to find the optimal (or near optimal) motifs. Many of these algorithms can be classified into one of two broad categories: stochastic searching algorithms based on position specific weight matrix (PSWM) motif representations, and combinatorial search algorithms based on consensus sequence motif representations. Examples of the first category include the well-known programs such as MEME [1], AlignACE [17], GibbsSampler [11], BioProspector [13], while the second category can be exemplified by Weeder [14], YMF [18], MultiProfiler [8], and Projection [2]. For surveys of the existing methods and assessments of their relative performance, see $[21,12,7]$. As expected, no single method stands out as the sole best. In fact, assessing the performances of these algorithms is a daunting task itself, and experiments have shown that the overall performance of motif finding algorithms is still quite low $[21,7]$. Nevertheless, there seems to be a slight advantage by combinatorial approaches $[21,12]$.

To test the capacity of various motif finding algorithms, Pevzner and Sze designed a set of challenging cases, the socalled $(l, d)$-motif, a set of DNA $l$-mers each of which differ from a common consensus sequence by exactly d mismatches [15]. The motifs were then embedded into some random DNA sequences and submitted to various motif finding algorithms. It has been shown that many stochastic searching algorithms fail to recover the embedded motifs even for biologically realistic choices of parameters (e.g. a set of $(15,4)$-motifs embedded in 20 sequences each with 600 bases) [15]. On the other hand, although combinatorial search algorithms have generally been shown to perform better in these challenging test cases, they typically resort to exhaustive enumeration of all or a large number of variants of consensus sequences, and are therefore limited 
to small data sets and short motifs only.

In this study, we propose a novel motif finding algorithm based on a population-based stochastic optimization technique called Particle Swarm Optimization (PSO) [5], which has been shown to be effective in optimizing difficult multidimensional problems in many fields. The naive PSO algorithm, however, can only be applied to continuous domains. The motif finding problem, which is essentially a multiple local sequence alignment problem, unfortunately, is discrete, as a slight shift of one sequence completely changes the alignment (See Section 3.2 for more discussions). To circumvent this problem, we break sequences into $l$-mers and develop a novel mapping scheme to convert the motif finding problem into a semi-continuous domain, and modified the update policy of the original PSO algorithm to solve the motif finding problem. We also propose several strategies for escaping from local optima, and determining the termination criteria automatically.

Compared to previous motif finding methods, our algorithm uses consensus as motif representation, thus avoiding many of the pitfalls associated with PSWM-based methods. On the other hand, PSO does not require exhaustive enumeration of consensus sequences, yet can still quickly converge to an optimal or almost optimal solution. Experimental results on both simulated and real biological data sets have shown that our method is more efficient and more accurate than several existing algorithms.

The remaining sections are organized as follows. In Section 2, we introduce the basic concept and ideas of PSO and the generic PSO algorithm. In Section 3, we discuss the improvement that we made to the generic PSO algorithm in order to accommodate the unique nature of motif finding problems, and other algorithmic issues that we addressed. We present our experimental results in Section 4, and conclude in Section 5 with some possible future improvement.

\section{Particle Swarm Optimization}

Particle Swarm Optimization (PSO) is a populationbased stochastic optimization technique for problem solving that is inspired by the social behaviors of organisms such as bird flocking and fish schooling [5]. The system is initialized with a population of random solutions and searches for the optimal solution by updating iteratively. Although PSO shares many similarities with evolutionary computation techniques such as genetic algorithms, PSO differs from other evolutionary algorithms significantly on how the solutions were updated. In PSO, each potential solution, called particle, is represented by a point in the multiple-dimensional solution space. When searching for the optimal solution, particles fly around the solution space with a certain velocity. During flight, each particle adjusts its position and velocity according to its own experience and the experiences of its neighbors. Specifically, each particle keeps track of the best solution (the position and the fitness value) it has encountered so far. This solution is called pbest, which stands for personal best. Each particle also keeps track of the best solution by any particle in its neighborhood. This solution is called lbest, which stands for local best. Many types of neighborhood structures can be implemented by emulating real social networks. In the simplest case, all the particles are directly connected to each other. Then lbest is simply the global optimum of all the particles, hence is also called gbest. Therefore, while each individual particle is performing a local search, the particles also communicate with other particles and learn from them, balancing exploration and exploitation.

Formally, let vectors $\mathbf{x}_{i}$ and $\mathbf{v}_{i}$ be the current position and velocity of the $i$-th particle $(1 \leq i \leq n), \widehat{\mathbf{x}}_{i}$ be the recorded position of pbest of the $i$-th particle, and $\widehat{\mathrm{g}}$ be the position of gbest. The fundamental concept of PSO consists of changing the velocity $\left(\mathbf{v}_{i}\right)$ of each particle at each time step toward its pbest and gbest locations. Acceleration is weighted by a random number, with separate random numbers generated for acceleration toward pbest and gbest locations. The particles update their positions and velocities based on the two equations below.

$$
\begin{aligned}
\mathbf{v}_{i} & =\omega \mathbf{v}_{i}+c_{1} \mathbf{r}_{1} \circ\left(\mathbf{x}_{i}-\widehat{\mathbf{x}}_{i}\right)+c_{2} \mathbf{r}_{2} \circ\left(\mathbf{x}_{i}-\widehat{\mathbf{g}}\right) ; \\
\mathbf{x}_{i} & =\mathbf{x}_{i}+\mathbf{v}_{i},
\end{aligned}
$$

where $\omega$ is a parameter called the inertial weight, $\mathbf{r}_{1}$ and $\mathbf{r}_{2}$ are vectors of random numbers, usually uniformly distributed within 0 and 1 . The operator $\circ$ denotes entry-wise vector multiplication. It is critical to note that different $\mathbf{r}_{1}$ and $\mathbf{r}_{2}$ are generated at each iteration and for each particle. $c_{1}$ and $c_{2}$ are positive constants, called the acceleration constant. $c_{1}$ is a factor determining how much the particle is influenced by its pbest, and $c_{2}$ is a factor determining how much the particle is influenced by gbest. Although the PSO algorithm is a relatively new technique, it has been developed into many variants and has been successfully applied in many research and application areas $[9,4,3,16]$.

However, as we have mentioned in Introduction, the PSO algorithm cannot be directly applied to the motif finding problem, which is a discrete optimization problem. In Section 3.2, we will discuss more in detail about the problems in applying PSO to motif finding, and our modifications of the naive algorithm to address these problems.

\section{The PSO-motif algorithm}

\subsection{Motif model and fitness function}

In order to apply PSO to the motif finding problem, we need to first define the solution structure and the fitness 
function. There are several ways to represent a motif. In PSWM-based algorithms, motifs are represented by weight matrices, so a solution can be unambiguously represented by a $3 \times l$ matrix, where $l$ is the length of the motif. Our focus of this paper is to develop a consensus-based algorithm, due to its relative advantage that has been shown recently $[21,12]$. To represent a motif in consensus-based algorithms, we have two choices. First, we may represent a motif by a consensus sequence, which is a discrete $l$-dimensional vector. The search space consists of all $4^{l}$ $l$-mers. Alternatively, a motif can be precisely defined by the list of motif instances occurred in the input sequences. Therefore, with the motif length fixed, a motif can be represented by a vector of motif starting positions within the input sequences. Between the two representations, we opt for the second representation in this work. The main advantage of the latter is its efficiency in updating. A more detailed discussion of this issue is beyond the scope of this paper.

To evaluate the quality of a motif, we first derive a consensus for the motif by taking the most frequent base at each position, and then measure the total number of mismatches between the individual instances and the consensus. When this fitness function is used, it is assumed that the background sequences have uniform base frequencies, which is usually not true. However, it is straightforward to design a more elaborated fitness function to take into consideration background base frequencies, with slightly increased running time.

\subsection{Word dissimilarity graph}

The main difficulty associated with applying the PSO algorithm to motif finding problem is that the fitness function is not continuous. In a typical PSO algorithm, one wishes to control the velocity so that at the beginning stage the particles can fly around quickly inside the search space, and when a particle approaches the optimal solution, it should slow down so it can converge. One can achieve this if the fitness function is continuous, since the velocity is updated according to the distances between the current position and the positions of pbest and gbest. However, a solution in our algorithm consists of a vector of positions in the input sequences. Therefore, the distance between two potential solutions has no indication of the difference of their fitness values. For example, as shown in the figure below, positions 1 and 2 are separated by 6 mismatches, while positions 1 and $\mathrm{N}$ have only 1 mismatch.

To solve this problem, we model the neighborhood information in the solution space by a dissimilarity graph of all $l$-mers in each sequence. For example, given an input sequence CTCTGCTG and motif length $=3$, we can construct a dissimilarity graph, whose adjacency matrix is shown in

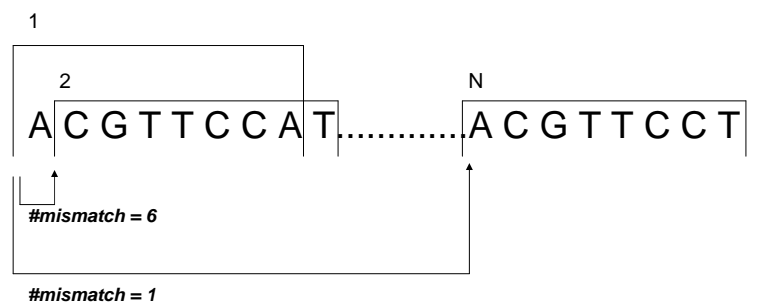

\section{Figure 1. Relationship between motif posi- tions and motif dissimilarity}

\section{Table 1. Adjacency matrix of an example dis- similarity graph}

\begin{tabular}{|c|c|c|c|c|c|c|}
\hline & P1 & P2 & P3 & P4 & P5 & P6 \\
\hline P1 & - & 3 & 1 & 2 & 3 & 1 \\
\hline P2 & 3 & - & 3 & 2 & 1 & 3 \\
\hline P3 & 1 & 3 & - & 3 & 3 & 0 \\
\hline P4 & 2 & 2 & 3 & - & 3 & 3 \\
\hline P5 & 3 & 1 & 3 & 3 & - & 3 \\
\hline P6 & 1 & 3 & 0 & 3 & 3 & - \\
\hline
\end{tabular}

Table 1. The row index is the current position of a candidate motif in this sequence, the column index is the potential new position of the motif, and the values in the cell are the number of mismatches between the two l-mers starting at the positions represented by the row index and column index, respectively. For example, if the motif start position is updated from 1 to 6 , the distance is only 1 , meaning CTC and CTG have 1 mismatch. With this graph, we can reformat the sequence into a new order, which is more meaningful and useful. In the matrix below, the "neighborhoods" order of $\mathrm{P} 2$ is (P5), (P4), (P1;P3;P6). This matrix can be pre-computed and stored in the main RAM. As one graph is needed for each sequence, the total space needed is $O\left(n L^{2}\right)$, where $n$ is the number of sequences and $L$ is the length of each sequence. For longer sequences, the graph can be made sparse by keeping only the edges whose weights are smaller than a certain threshold, for example, $2 d$, where $d$ is the maximum number of mismatches expected between the motif consensus and its instances.

\subsection{Update policy}

In order to decide what new positions should be considered as the motif starting positions, we define the velocity of a particle as the range of the allowed number of mismatches between the new and current motif instances. Let $\mathbf{x}_{i}$ and $\mathbf{x}_{j}$ represent two potential solutions, and $\mathbf{D}\left(\mathbf{x}_{i}, \mathbf{x}_{j}\right)$ be the vector of numbers of mismatches between the motif instances in $\mathbf{x}_{i}$ and in $\mathbf{x}_{j}$, we use the following rule to define 
the velocity of a particle:

$$
\begin{gathered}
\mathbf{v}_{i}^{u} \leftarrow \omega \mathbf{v}_{i}^{u}+c_{1}^{u} \mathbf{r}_{1} \circ \mathbf{D}\left(\mathbf{x}_{i}, \widehat{\mathbf{x}}_{i}\right)+c_{2}^{u} \mathbf{r}_{2} \circ \mathbf{D}\left(\mathbf{x}_{i}, \mathbf{g}\right) \\
\mathbf{v}_{i}^{l} \leftarrow \omega \mathbf{v}_{i}^{l}+c_{1}^{l} \mathbf{r}_{1} \circ \mathbf{D}\left(\mathbf{x}_{i}, \widehat{\mathbf{x}}_{i}\right)+c_{2}^{l} \mathbf{r}_{2} \circ \mathbf{D}\left(\mathbf{x}_{i}, \mathbf{g}\right)
\end{gathered}
$$

Given the upper and lower bounds of the velocity, $\mathbf{v}_{i}^{u}$ and $\mathbf{v}_{i}^{l}$, we update $\mathbf{x}_{i}$ as follows. Let $x_{i}(j)$ be the starting position of the motif on the $j$-th sequence, and $x_{i}^{\prime}(j)$ be the new starting position on the same sequence. In order to obtain $x_{i}^{\prime}(j)$, we randomly pick a position from sequence $j$ such that the number of mismatches between the motifs started at these two positions are within the upper and lower bound of the velocity, i.e.,

$$
v_{i}^{l}(j) \leq D\left(x_{i}(i), x_{i}^{\prime}(j)\right) \leq v_{i}^{l}(j) .
$$

\subsection{Occasional full scan and shift check}

Inside the algorithm we always keep track a consensus sequence derived from gbest using the most frequent base at each position, and compute the fitness value of gbest. When the fitness value of gbest reaches a certain threshold, we use the consensus sequence to do a full scan on all sequences to check if there is an $l$-mer in the input sequence that matches the consensus sequence better than the $l$-mer in gbest.

Second, similar to many motif finding algorithms, the output of PSO algorithm may have a shift issue: the start positions may be one or two positions away from the real positions, and it is difficult for the algorithm to escape from such local optima. To circumvent this problem, we periodically check whether shifting the motif positions by a small number can improve the quality of the solution.

When a particle is almost near the optimal solution, these two tricks can significantly reduce the running time, and make the algorithm converge much more quickly.

\subsection{Escape from local optima}

If gbest remains stable for a large number of iterations, we consider that the algorithm has reached a local optimum or the global optimum, so we reset the algorithm. There are two ways for reset. The first strategy is to simply discard all the information so far and start the algorithm from scratch. In some cases with this strategy the algorithm might converge to the same local optimum repeatedly. Here we suggest the second strategy, which we call a "reset move". When a local optimum is detected, we move all the current solution, pbest and gbest by a random distance. We have found that this strategy can more effectively help the algorithm escape from local optima.

\subsection{Termination}

Like many stochastic algorithms, in many cases it is difficult to determine when the algorithm should terminate. For simulated test cases, we typically know the total number of mismatches, so the program can stop when it reaches the threshold. In practice, however, a user typically does not have prior knowledge about the number of mismatches. If the number is given too high, the algorithm may give up the search before it finds the optimal solution. On the other hand, if the number is given too low, the algorithm may spend most of its time trying to improve over an easily detectable global optimum. Here we implemented three methods to determine when the algorithm should terminate: value-based, time-based, and repeat-based. The valuebased method is the easiest: when the solution reaches a pre-defined threshold value, the algorithm stops running and returns the solution. The time-based method terminates the algorithm after a certain amount of time has passed. Finally, we recommend the repeat-based method for real test cases. With this method, before resetting the algorithm, we compare the current gbest fitness value with the best gbest fitness value achieved during the course of the algorithm. If the current gbest is better than the history gbest, we update the history gbest; if the two fitness values and the associated motif consensuses are exactly the same, we stop the algorithm and output this result. This method is based on the intuition that, since there are typically many local optima, the probability of repeating one local optimum without an update is very low. This method is very effective when tested on the real test cases (Section 4).

\subsection{Post-processing}

By default, the quality of a motif is evaluated by the total number of mismatches between the consensus sequence and its instances. There are a number of limitations in this basic strategy when applied to real biological sequences. First, the mismatch based quality function does not consider the background frequency, which is usually not uniform. Second and more importantly, in real TF binding motifs the mutation rates are often not uniformly distributed across every position, and not every site has the same significance in determining the binding strength. For example, many TF binding sites consist of two short conserved regions, separated by a small gap. Therefore, a mismatch in the gapped region should be penalized much less than a mismatch in the conserved region. However, in the consensusbased method, we are forced to select a representative for each position, and the number of mismatches is computed based on that representative.

To address these problems, we apply a post-processing procedure to improve the final motif returned by the PSO 


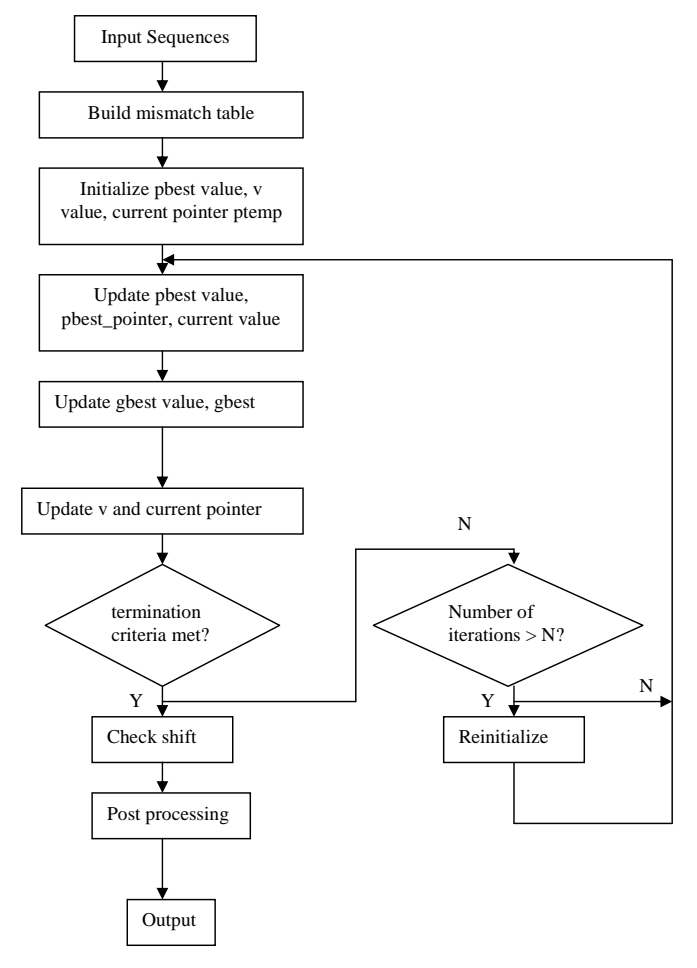

Figure 2. Overview of PSO-motif algorithm

algorithm. Given the gbest returned by the algorithm, we construct a position-specific weight matrix. This matrix is then used to scan all input sequences. This scan will likely update some of the motif instances. We then recompute the position specific weight matrix and repeat the scan, until the solution does not vary. With this method, the matching/mismatching score is weighted by the information content contained in each position, and therefore the more conserved positions will have more contribution to the selection of the binding sites. Furthermore, we can also take into account the background base frequencies when scoring a binding site against the weight matrix.

Figure 2 shows the overall structure of the PSO-motif algorithm. The individual steps have been described in the previous subsections.

\section{Experimental results}

We have implemented our algorithm in C. To evaluate the performance of our algorithm, we tested it on two types of DNA sequences. The first type of test data consists of simulated data sequences, also known as the $(l, d)$-motif challenging problem. The second type of data contain real promoter sequences from $E$. coli and human genes containing known TF binding sites that have been determined experimentally.

\subsection{Simulated data sets}

To objectively compare with the existing algorithms, we first tested our algorithm on simulated data sets. We synthesized problem instances as follows. First, a motif consensus of length $l$ was generated by randomly picking $l$ bases. Second, we randomly selected $d$ positions from the consensus and mutated the base at each position to a random base, which could be the same as the original base. This generates one instance of the motif. We repeated this process to obtain $t$ instances. Third, we randomly generated $t$ background sequences of length $n$ each. Finally, we assign each motif instance to a random position in a background sequence. This procedure generates $t$ sequences, each containing exactly one instance of the motif. All random choices were made independently and with equal base frequencies.

We first focused on the $(15,4)$-motif challenge problem, which is one of the most popular benchmarks for many motif finding programs. We fixed the number of sequences to 20 , and varied the length of each sequence from 400 to 1000. Since our algorithm is stochastic, we repeated our algorithm multiple times, and terminated it once the motif was found. Table 2 shows the mean and median running time of our algorithm. For each sequence length, the results are based on 10 independent runs on 5 sets of sequences. Table 2 also shows the running time of two of the best combinatorial-search motif finding algorithms: Projection [2], a random projection based algorithm, and Weeder, a suffix tree based enumeration algorithm [14]. The running time of Weeder was taken from the original paper published almost 7 years ago [14]. According to the original authors, the running time was based on an $89 \%$ success probability. Unfortunately we were not able to repeat the experiments ourselves since the program is not available. The program Projection was downloaded from the original author's website (http://cse.wustl.edu/ jbuhler) and we tested the program on the same data sets as for our algorithm. The number of iterations of Projection is pre-determined by its parameters, so its running time does not vary between runs and the mean is almost the same as the median. Therefore only the mean results are shown for Projection. PSO-motif and Projection were able to recover all the embedded motifs with $100 \%$ accuracy. Weeder is generally slower than Projection and PSO-motif. While PSO-motif and Projection have similar running time, the running time of Projection increases more rapidly with the length of the input sequences. Furthermore, the number of iterations in Projection has to be estimated from the motif length and the number of mismatches in advance. If a user does not know the number of mismatches, one either has to use a large number of iterations which requires a prolonged running time, or use a small number of iterations with the risk of missing the real motif. 


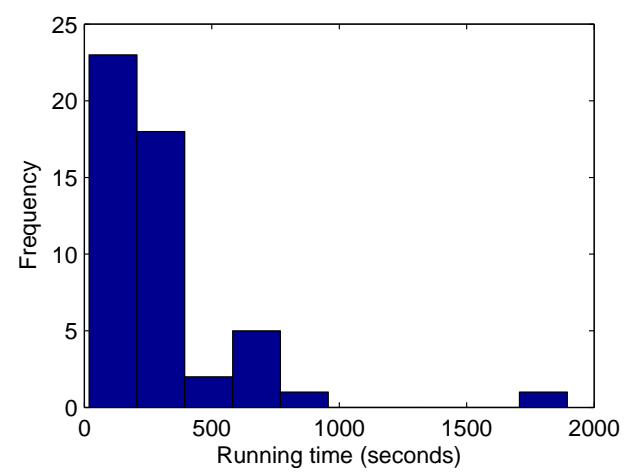

Figure 3. Distribution of running time of PSO on $(15,4)$ motifs with sequence length $=1000$.

It is also worth noting that since PSO is a stochastic algorithm, its running time varies between runs even on the same data set. In rare cases the algorithm may require extremely long running time to converge. As a result, the running time has a long tail distribution, and the mean is often much higher than the median. Figure 3 shows the distribution of the running time of PSO on $(15,4)$ motifs with a sequence length of 1000 . In fact, in $80 \%$ of cases the motifs can be found in 335 seconds, while in one case the program completes after almost 1900 seconds. A better strategy for detecting local optima may eliminate some of these rare cases and improve the efficiency.

Next, we compared PSO, Projection and MotifEnumerator, a pattern-driven motif enumeration algorithm [20], on a series of challenge problems with varying motif lengths and number of errors. Sequences lengths are fixed at 600. The program MotifEnumerator was downloaded from the original author's website (http://faculty.cs.tamu.edu/shsze/motifenumerator/). Again both PSO-motif and Projection were able to recover the embedded motifs with $100 \%$ accuracy. As shown in Table 3, although Projection is more efficient than PSO for shorter motifs, its advantage vanishes when motif length increases to about 15, and it is slower than PSO for motifs longer than 17. The running time of PSO is almost independent of motif lengths. The running time and space requirement of MotifEnumerator are exponential to $l$. Therefore, for small motif lengths (e.g., $l=11$ ), MotifEnumerator solves the problem very efficiently; however, when $l$ increases to 15 , the program aborted with an out of memory exception on our testing computer with 2GB RAM.

\subsection{Real biological sequences}

We also tested our algorithm on three sets of biological sequences with known TF binding motifs. (1) The first
Table 2. Running time on $(15,4)$-motif challenge problems

\begin{tabular}{l|r|r|r|r|r}
\hline Sequence length & 400 & 500 & 600 & 800 & 1000 \\
\hline Weeder & $<1 \mathrm{~m}$ & $125 \mathrm{~s}$ & $200 \mathrm{~s}$ & $450 \mathrm{~s}$ & $15 \mathrm{~m}$ \\
\hline Projection & $9 \mathrm{~s}$ & $23 \mathrm{~s}$ & $42 \mathrm{~s}$ & $162 \mathrm{~s}$ & $418 \mathrm{~s}$ \\
\hline PSO (mean) & $18 \mathrm{~s}$ & $34 \mathrm{~s}$ & $57 \mathrm{~s}$ & $137 \mathrm{~s}$ & $288 \mathrm{~s}$ \\
\hline PSO (median) & $7 \mathrm{~s}$ & $15 \mathrm{~s}$ & $36 \mathrm{~s}$ & $103 \mathrm{~s}$ & $220 \mathrm{~s}$ \\
\hline
\end{tabular}

Table 3. Running time on other motif challenge problems

\begin{tabular}{l|r|r|r|r|r}
\hline$(l, d)$ & $(11,2)$ & $(13,3)$ & $(15,4)$ & $(17,5)$ & $(19,6)$ \\
\hline Projection & $4 \mathrm{~s}$ & $13 \mathrm{~s}$ & $42 \mathrm{~s}$ & $94 \mathrm{~s}$ & $174 \mathrm{~s}$ \\
\hline MotifEnumerato & $5 \mathrm{~s}$ & $119 \mathrm{~s}$ & - & - & - \\
\hline PSO (mean) & $72 \mathrm{~s}$ & $58 \mathrm{~s}$ & $57 \mathrm{~s}$ & $61 \mathrm{~s}$ & $54 \mathrm{~s}$ \\
\hline PSO (median) & $43 \mathrm{~s}$ & $48 \mathrm{~s}$ & $36 \mathrm{~s}$ & $38 \mathrm{~s}$ & $41 \mathrm{~s}$ \\
\hline
\end{tabular}

sample is the binding sites for the cyclic AMP receptor protein (CRP), which functions as a transcription factor in Escherichia coli. The data set contains 18 sequences, each 105 bp long, which contain 23 sites that have been experimentally determined [19]. (2) The second test sample is the binding site for the estrogen receptor (ER), which is a ligand-activated enhancer protein that binds to estrogen response elements (EREs).The data set includes 25 genomic sequences, each of which is $200 \mathrm{bp}$ long and contains a single known ERE [10]. (3) The final data set includes 25 mammalian sequences of $200 \mathrm{bp}$ long that are known to contain binding sites for the transcription factors in the E2F family [6].

It is important to note the following difference between the above sequences and the simulated ones. (1) The background base frequencies are not uniform in these real sequences; (2) The number of mutations are not known; and (3) The mutation rates vary at different positions. It is interesting to test whether any of these characteristics in real sequences may pose additional difficulty for our consensusbased motif finding algorithm. Furthermore, we chose these three examples because they represent three typical cases in motif finding: ERP is a long and well-conserved prokaryotic TF binding site, which is relatively easy to be identified; ERE consists of two conserved sub-motifs separated by a small gap; E2F is a mammalian TF binding motif present in promoter sequences with high GC contents which may mislead an algorithm into a low complexity region.

Figure 4 shows the sequence logos of the known motifs together with the flanking regions, compared with the motifs predicted by our algorithm. As shown, our algorithm has recovered the consensuses of all three real motifs. On 

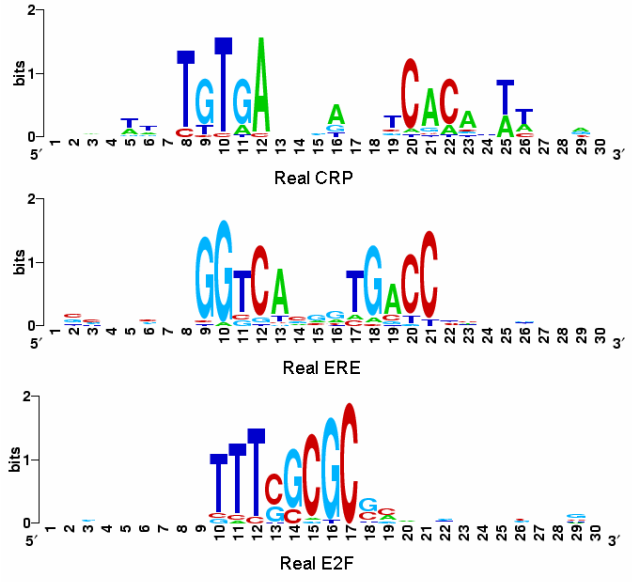

(a) Real motifs
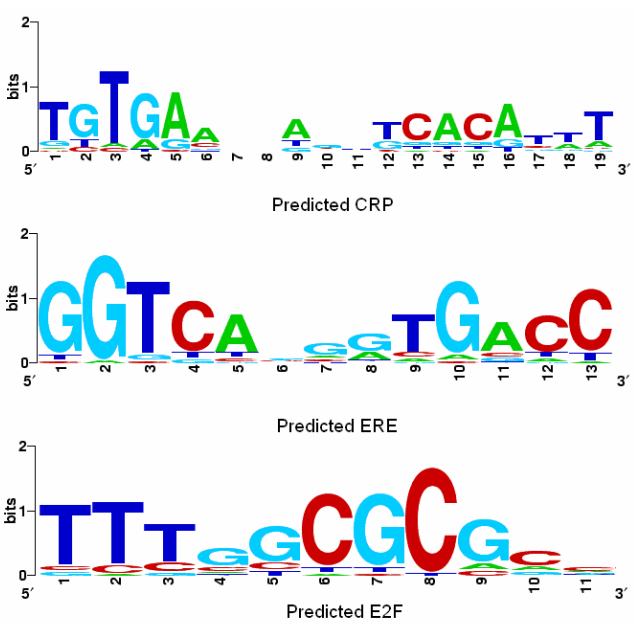

(b) Predicted motifs

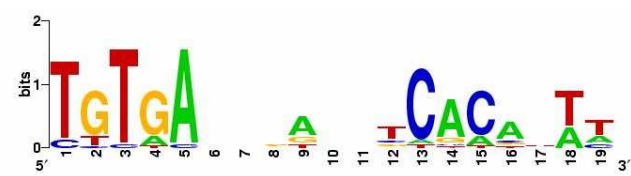

Postprcessed CRP
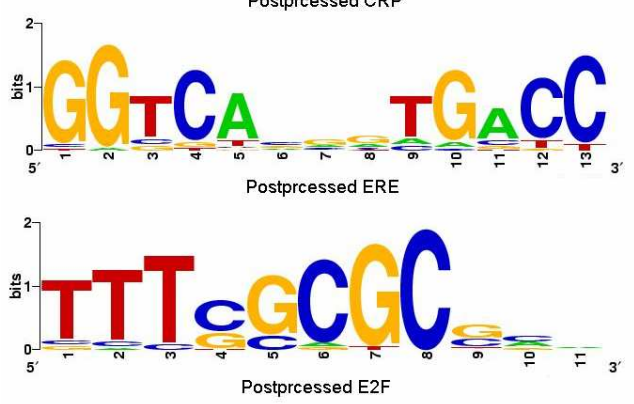

(c) Predicted motifs after post-processing

Figure 4. (color online) Results of PSO-motif on real biological sequences.

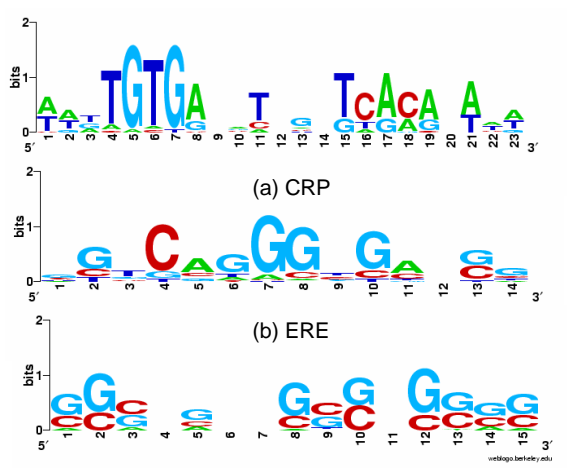

(c) E2F

Figure 5. (color online) Results of alignACE.
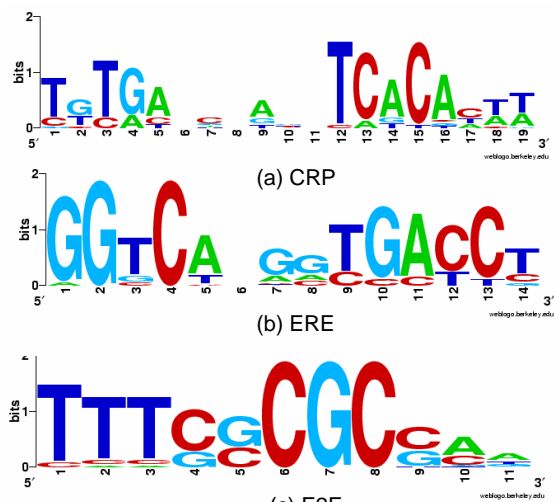

(c) E2F

Figure 6. (color online) Results of MEME.

the other hand, the probabilities of observing a given base at some position are different between the predicted and the real motifs, especially for the CRP motif. Further investigation shows that the reason for this partial discrepancy is often due to (1) each input sequence may contain more than one motif, while our algorithm only allowed one occurrence per sequence; (2) our algorithm does not take into consideration background base frequencies. In the CRP data set, the 18 sequences contained a total of 24 binding sites. Furthermore, the sequences contain a very high AT content (61\%). Our algorithm included several binding sites that match to the consensus sequence equally well as or better than the true sites, but have higher AT contents. Therefore, although the binding sites identified by our algorithm has a smaller number of mismatches to the consensus than the real binding sites, the former may be less biologically interesting. Therefore, we applied to post-processing procedure described in Section 3.7. Figure 4(c) shows that the post-processing did not change the consensus sequence, but fine-tuned the motif instances and the predicted motifs with post-processing are more similar to the real ones than those without post-processing are. 
As a comparison, we applied two of the most successful motif finding algorithms, MEME [1] and AlignACE [17], both of which are based on optimizing position specific weight matrices. To our surprise, even for these relatively easy problems, AlignACE failed to find the ERE and E2F motifs. Although it found the consensus sequence of CRP, it missed many of the actual binding sites (Fig. 5) . MEME correctly identified all the consensus sequences, but still made significant number of errors on the binding site level (Fig. 6) . These results confirmed the weakness of PSWMbased methods $[21,12]$.

\section{Conclusions and discussion}

In this work, we have proposed a novel algorithm for finding DNA motifs based on a modified version of the Particle Swarm Optimization (PSO) algorithm. We have shown that by remapping a promoter sequence into a dissimilarity graph of 1-mers, we can successfully apply the PSO algorithm to solve the difficult motif finding problem with high efficiency and high accuracy. Our experimental results on both simulated and real biological data sets are very encouraging. When applied to simulated challenge problems, PSO is slower than Projection in easier cases, while faster in more difficult cases that have longer input sequences or longer motifs and more mutations. Furthermore, our algorithm does not require the number of mismatches to be given as a parameter, which is more useful in practice. For real biological sequences, our method combined with postprocessing has successfully identified the known motifs and most of the binding sites. Our studies have shown that PSO is a reliable and efficient technique for solving the difficult motif-finding problem, and we are looking into applying it to other challenging problems in computational biology.

Acknowledgement JR was supported in part by a UTSA new faculty startup grant and a faculty research award. The authors would like to thank Zhi Wei for providing real biological sequence data, and UTSA Computational Biology Initiative for providing computing resources.

\section{References}

[1] T. Bailey and C. Elkan. Fitting a mixture model by expectation maximization to discover motifs in biopolymers. Proc Int Conf Intell Syst Mol Biol, 2:28-36, 1994.

[2] J. Buhler and M. Tompa. Finding motifs using random projections. J Comput Biol, 9:225-42, 2002.

[3] M. Clerc and J. Kennedy. The particle swarm-explosion, stability, and convergence in a multidimensional complex space. IEEE Transactions on antennas and propagation., 6:58-73, 2002.

[4] R. Eberhart and Y. Shi. Particle swarm optimization: developments, applications and resources. In Proc. 2001 Congr. Evolutionary Computation, 2001.
[5] R. Eberhart, Y. Shi, and J. Kennedy. Swarm Intelligence. Morgan Kaufmann, 2001.

[6] M. Frith, U. Hansen, J. Spouge, and Z. Weng. Finding functional sequence elements by multiple local alignment . $\mathrm{Nu}$ cleic Acids Res., 32:189-200, 2004.

[7] J. Hu, B. Li, and D. Kihara. Limitations and potentials of current motif discovery algorithms. Nucleic Acids Res., 33(15):4899-913, 2005.

[8] U. Keich and P. Pevzner. Finding motifs in the twilight zone. Bioinformatics, 18:1374-81, 2002.

[9] J. Kennedy and R. Eberhart. Particle swarm optimization. In Proc. IEEE Conf. Neural Networks IV, 1995.

[10] C. Klinge. Estrogen receptor interaction with estrogen response elements. Nucleic Acids Res., 29:2905-2919, 2001.

[11] C. Lawrence, S. Altschul, M. Boguski, J. Liu, A. Neuwald, and J. Wootton. Detecting subtle sequence signals: a gibbs sampling strategy for multiple alignment. Science, 262:20814, 1993.

[12] N. Li and M. Tompa. Analysis of computational approaches for motif discovery. Algorithms Mol Biol., 1:8, 2006.

[13] X. Liu, D. Brutlag, and J. Liu. Bioprospector: discovering conserved dna motifs in upstream regulatory regions of co-expressed genes. Pac Symp Biocomput., pages 127-38, 2001.

[14] G. Pavesi, G. Mauri, and G. Pesole. An algorithm for finding signals of unknown length in dna sequences.. Bioinformatics, 17:S207-14, 2001.

[15] P. Pevzner and S. Sze. Combinatorial approaches to finding subtle signals in dna sequences. Proc Int Conf Intell Syst Mol Biol., 8:269-78, 2000.

[16] J. Robinson, S. Sinton, and Y. Rahmat-Samii. Particle swarm optimization in electromagnetics. IEEE Transactions on antennas and propagation., 52:397-407, 2004.

[17] F. Roth, J. Hughes, P. Estep, and G. Church. Finding DNA regulatory motifs within unaligned noncoding sequences clustered by whole-genome mrna quantitation. Nat Biotechnol., 16:939-45, 1998.

[18] S. Sinha and M. Tompa. Discovery of novel transcription factor binding sites by statistical overrepresentation. Nucleic Acids Res., 30:5549-60, 2002.

[19] G. Stormo and G. Hartzell. Identifying protein-binding sites from unaligned dna fragments. Proc. Natl Acad. Sci., 86:1183-1187, 1989.

[20] S.-H. Sze and X. Zhao. Improved pattern-driven algorithms for motif finding in dna sequences. In Proc. of the 2005 Joint RECOMB Satellite Workshops on Systems Biology and Regulatory Genomics, Lecture Notes in Bioinformatics, volume 4023, pages 198-211, 2006.

[21] M. Tompa, N. Li, T. Bailey, G. Church, B. De Moor, E. Eskin, A. Favorov, M. Frith, Y. Fu, W. Kent, V. Makeev, A. Mironov, W. Noble, G. Pavesi, G. Pesole, M. Rgnier, N. Simonis, S. Sinha, G. Thijs, J. van Helden, M. Vandenbogaert, Z. Weng, C. Workman, C. Ye, and Z. Zhu. Assessing computational tools for the discovery of transcription factor binding sites. Nat Biotechnol, 23:137-44, 2005. 